www.jmscr.igmpublication.org Impact Factor 5.244

Index Copernicus Value: 83.27 ISSN (e)-2347-176x ISSN (p) 2455-0450 crossref DOI: _https://dx.doi.org/10.18535/jmscr/v4i12.82

\title{
Partial Trisomy 18 in a Newborn Born to Retro Positive Parents- A Case Report
}

\author{
Authors \\ Dr Rohit Kapoor, Dr Souvik Guha, Dr Garima Biyani, Dr Hriday De, \\ Dr Syed Md Azad \\ ICH, Kolkata INDIA
}

\begin{abstract}
The patient was a dysmorphic newborn born to retropositive parents while the mother was on ART and needed resuscitation after birth. The child was lethargic since birth and had respiratory distress. Investigations revealed multiple congenital anomalies. A karyotyping was done which revealed a translocation between the long arm of chromosomes 18 and 22 with partial trisomy of chromosome 18.
\end{abstract}

\section{INTRODUCTION}

The trisomy 18 syndrome, also known as Edwards syndrome, is a common chromosomal disorder due to the presence of an extra chromosome 18 , either full, mosaic trisomy, or partial trisomy $18 \mathrm{q}$. The condition is the second most common autosomal trisomy syndrome after trisomy 21 . The live born prevalence is estimated at $1 / 6,000$ $1 / 8,000$, but the overall prevalence is higher (1/2500-1/2600) due to the high frequency of fetal loss and pregnancy termination after prenatal diagnosis. The prevalence at birth is higher in females compared to males ( $\mathrm{F}$ : M \%, 60.4), but this discordance is not present if the sex ratio is calculated among fetuses electively terminated (F:M \% 48:51.) ${ }^{1}$. The prevalence of trisomy 18 rises with the increasing maternal age. The recurrence risk for a family with a child with full trisomy 18 is about $1 \%$. The recognizable syndrome pattern consists of major and minor anomalies, prenatal and postnatal growth deficiency, an increased risk of neonatal and infant mortality, and marked psychomotor and cognitive disability. Typical minor anomalies include characteristic craniofacial features, clenched fist with overriding fingers, small fingernails, underdeveloped thumbs, and short sternum. The presence of major malformations is common, and the most frequent are heart and kidney anomalies. Feeding problems occur consistently and may require enteral nutrition. The major causes of death include central apnea, cardiac failure due to cardiac malformations, respiratory insufficiency due to hypoventilation, aspiration, or upper airway obstruction and, likely, the combination of these and other factors.

\section{CASE PRESENTATION}

Term, small for gestational age, birth weight$2.01 \mathrm{~kg}$, male child born out of LUCS at 37 weeks gestation, did not cry after birth and required bag and mask ventilation for 60 seconds. Apgar score at 1 and 5 minute was 5 and 8 respectively. The child was born to retropositive parents and the 


\section{JMSCR Vol||04||Issue||12||Page 14819-14822||December}

mother was on ART since the first trimester. The viral load prior to delivery was $<90$ copies $/ \mathrm{ml}$. On examination the baby was lethargic with poor cry and tone. He had a dysmorphic facies with microretrognathia, wide prominent metopic suture and mid facial hypoplasia (FIG 1). Head was dolicocephalic and head circumference was $33.5 \mathrm{~cm}$. Rib cage was dystrophic and CVS examination revealed a grade IV pansystolic murmur at the left sternal border with a loud P2. He had short stubby fingers. There were no other abnormalities in the systemic examination. Post resuscitation the child was having respiratory distress and was given 2 liters moist oxygen by hood. The child was started on syrup zidovudine soon after birth. CXR showed cardiomegaly with dystrophic chest wall and patchy opacity in the right lower lung field (FIG 2). The baby continued to have subcoastal and intercoastal suction due to dystrophic chest wall even on day 2 without any oxygen requirement. The child was unable to suck and was started on nasogastric tube feeding. The child was started on IV antibiotics which were stopped after a sterile BACTEC. ECHO revealed tetralogy of fallot with right sided aortic arch, mild AR, PS gradient at birth was 40 and 70 at day 7 of life. USG KUB and Brain were normal. A RNA PCR viral load and TORCH screening was done which came out to be negative. The child had an episode of convulsion on day 9 and was started on IV phenobarbitone.MRI brain revealed MRI brain revealed abnormal signal intensities in bilateral frontal and periventricular region with dilated ventricles- ?due to perinatal infection/ hypoxic ischemic injury(FIG 3,4). Repeat CXR showed persistence of the right lower lobe opacity, so MRI chest was done which showed shows focal herniation of liver into the thoracic cavity in the right anterior retrosternal region likely representing Morgagni hernia/ focal eventration (FIG 5). Multiple system involvement prompted a karyotyping analysis which revealed a translocation between the long arm of chromosomes 18 and 22 with partial trisomy of chromosome 18 \{46XY der $(22) \mathrm{t}(18,22)$ (q11.2 $q 10),+18\}$ (FIG 6). The condition of the child gradually improved and full feed through NG tube was established and the child was discharged in a stable condition.

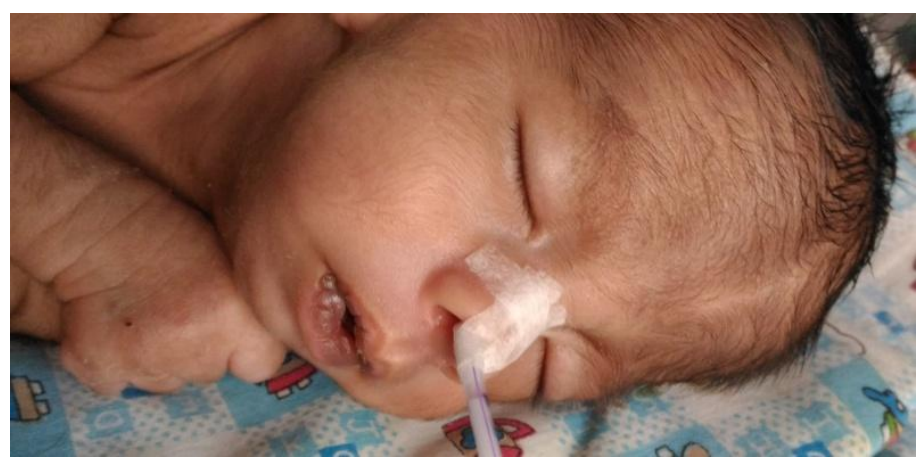

Figure 1

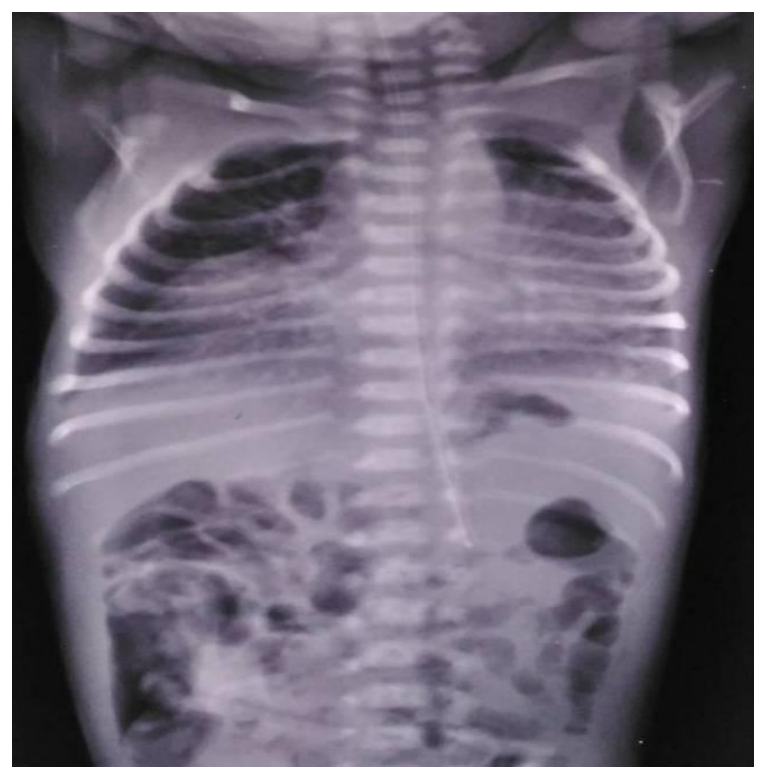

Figure 2

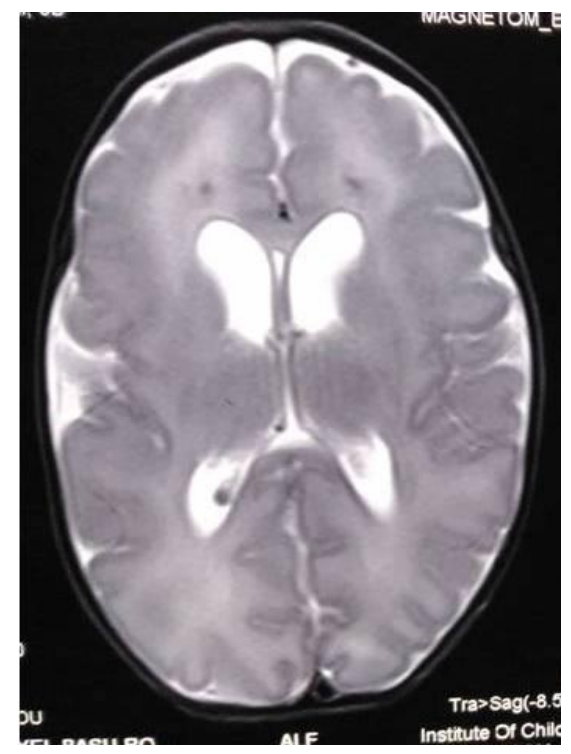

Figure 3 


\section{JMSCR Vol||04||Issue||12||Page 14819-14822||December}

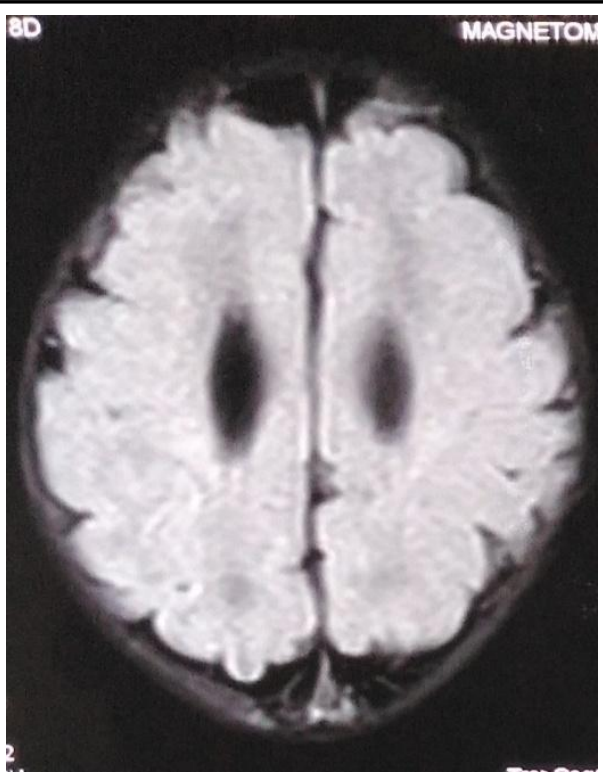

Figure 4

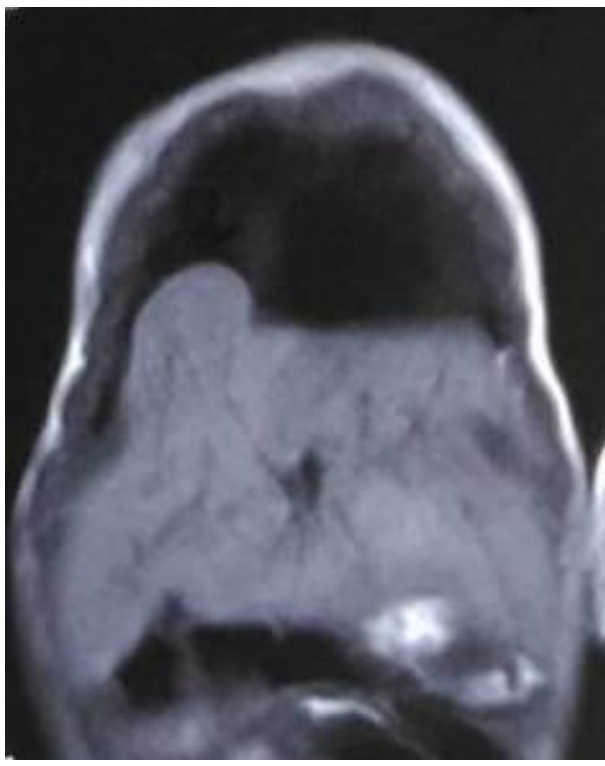

Figure 5

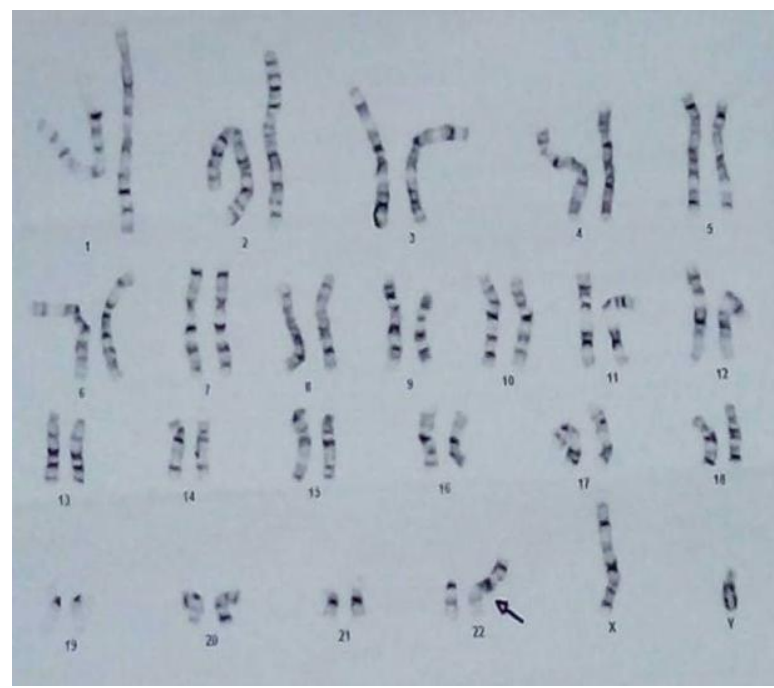

Figure 6

\section{DISCUSSION}

The trisomy 18 syndrome, also known as Edwards syndrome, is a common autosomal chromosomal disorder due to the presence of an extra chromosome 18. The first reported infants were described in 1960 by Edwards et al. and Smith et $\mathrm{al}^{2,3}$. The syndrome pattern comprises a recognizable pattern of major and minor anomalies, an increased risk of neonatal and infant mortality, and significant psychomotor and cognitive disability. The main clinical features represent the clues for the diagnosis in the perinatal period and include prenatal growth deficiency, characteristic craniofacial features, distinctive hand posture, nail hypoplasia, short hallux, short sternum, and major malformations (particularly involving the heart). The demonstration of an extra chromosome 18 , or less commonly a partial trisomy of the long arm of chromosome 18, on the standard G-banded karyotype allows for confirmation of the clinical diagnosis.

The trisomy 18 (or Edwards syndrome) phenotype results from full, mosaic, or partial trisomy $18 \mathrm{q}^{4}$.Complete or full trisomy 18 is the most common form (about 94\% of cases) whereas partial trisomy as found in our case is extremely rare. In the partial trisomy form only a segment of the chromosome 18 long arm is present in triplicate, often resulting from a balanced translocation or inversion carried by one parent. This type of trisomy accounts for approximately $2 \%$ of cases presenting with the Edwards phenotype. The location and the extent of the triplicated segment and the possible associated deletion of genomic material due to unbalanced translocation can explain the variable phenotype associated with partial trisomy ${ }^{5}$

The first study about postnatal survival of children with trisomy 18 was published in 1967: Weber reported a mean survival of 70 days $^{6}$. Most of the ensuing population studies showed a shorter survival, likely because, with prenatal and neonatal diagnosis, it is now possible to diagnose many cases, which would have died prior to detection in the past ${ }^{7}$. 


\section{REFRENCES}

1. Crider KS, Olney RS, Cragan JD: Trisomies 13 and 18: population prevalences, characteristics, and prenatal diagnosis, metropolitan Atlanta, 19942003. Am J Med Genet. 2008, 146A: 820826. 10.1002/ajmg.a.32200.

2. Edwards JH, Harnden DG, Cameron AH, Crosse VM, Wolff $\mathrm{OH}$ : A new trisomic syndrome. Lancet. 1960, 1: 787-789.

3. Smith DW, Patau K, Therman E, Inhorn SL: A new autosomal trisomy syndrome: multiple congenital anomalies caused by an extra chromosome. J Pediatr. 1960, 57: 338-345. 10.1016/S0022-3476(60)802417.

4. Embleton ND, Wyllie JP, Wright MJ, Burn J, Hunter S: Natural history of trisomy 18. Arch Dis Child. 1996, 75: 3841.

5. Carey JC: Trisomy 18 and trisomy 13 syndromes. Management of genetic syndromes 3rd edition. Edited by: Cassidy SB, Allanson JE. John Wiley \& Sons, New York; 2010:807-823.

6. Weber WW: Survival and the sex ratio in trisomy 17-18. Am J Hum Genet. 1967, 19: 369-377.

7. Root S, Carey JC: Survival in trisomy 18. Am J Med Genet. 1994, 49: 170-174. 10.1002/ajmg.1320490203. 